\title{
Indirect Sinus Lift with Implant Placement in Maxillary Premolar Region
}

\section{Priya S Reddy}

\section{ABSTRACT}

The maxilla is made up of spongy bone and has one of the least dense bones in oral cavity. Periodontal disease-stimulated teeth loss causes accentuated bone deficiency, both in height and in width by significant resorption of the alveolar bone. Bone remodeling in the region is further complicated by postextraction bone resorption, pneumatization of maxillary sinus, and poor quality of residual alveolar bone.

Indirect sinus augmentation is an effective solution for this problem. This case report presents the rehabilitation of maxillary premolar by using indirect sinus lift with implant placement where the bone height and bone width was compromised.

Keywords: Implant placement posterior maxilla, Indirect sinus lift, Maxillary sinus.

How to cite this article: Reddy PS. Indirect Sinus Lift with Implant Placement in Maxillary Premolar Region. J Health Sci Res 2016;7(1):32-34

\section{Source of support: Nil}

Conflict of interest: None

\section{INTRODUCTION}

An important requirement for the correct placement of the implant is the presence of an adequate quantity and quality of residual bone. ${ }^{1}$ Dental implants are used to replace both the form and the function of missing teeth. The presence of inadequate bone quality and quantity often complicates implant placement in posterior maxilla. A common problem encountered while placing implant in the posterior maxilla region is lack of adequate bone height required for successful implant therapy. Sinus lift procedures are the treatment of choice. The most widely used approaches for sinus lifting are direct and indirect.

Indirect sinus floor elevation technique is the preferred one. This case report presents the rehabilitation of the maxillary left premolar region with reduced bone height and width using indirect sinus lift procedure.

\section{Senior Lecturer}

Department of Periodontics, RajaRajeshwari Dental College \& Hospital, Bengaluru, Karnataka, India

Corresponding Author: Priya S Reddy, Senior Lecturer Department of Periodontics, RajaRajeshwari Dental College \& Hospital, Bengaluru, Karnataka, India, Phone: +918028437150 e-mail: subash_dr@rediffmail.com

\section{CASE REPORT}

A 28-year-old female patient reported to the Oxford Dental College, Bengaluru, with the chief complaint of missing teeth in upper and lower arches (Fig. 1).

A thorough clinical examination was done, followed by radiographic examination.

Various treatment options, such as removable partial denture (RPD), fixed partial denture (FPD) and implant were informed to the patient. The patient expressed her desire for the placement of implant. On evaluation of orthopantamograph (OPG) and cone beam computed tomography (CBCT), it was found that the left premolar region had only $6.5 \mathrm{~mm}$ of bone height, and an indirect sinus lift procedure was planned.

\section{SURGICAL PHASE}

- Implant selected was of $3.5 \mathrm{~mm}$ diameter and $9 \mathrm{~mm}$ length.

- Local anesthesia was given and crestal incision was made using no 15 BP blade (Fig. 2).

- Flap was reflected and it was found that the bone width was less in the maxillary left first premolar region (Fig. 3).

- Initial depth was achieved using a 2-mm pilot drill at a speed of 850 RPM with saline irrigation (Fig. 4).
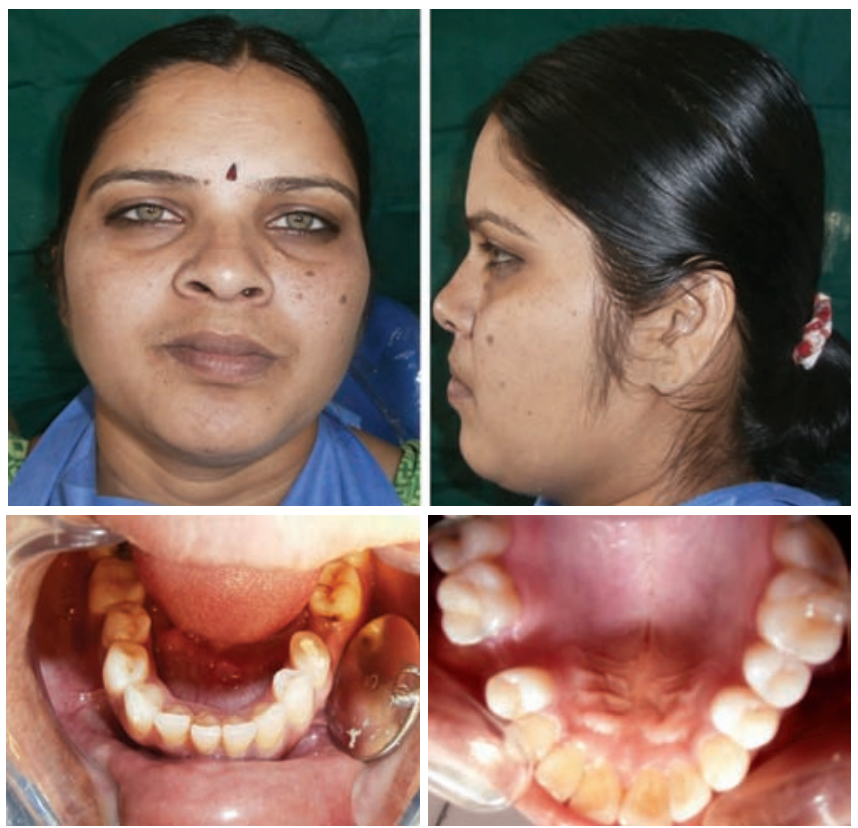

Fig. 1: Preoperative photographs 
- Indirect sinus lifting was done using sinus elevation osteotomes of various gauges from 2.7, extended up to 3.2 into the sinus floor (Fig. 5).

Gentle tap was given with a mallet on sinus lift osteotome to lift the sinus floor.

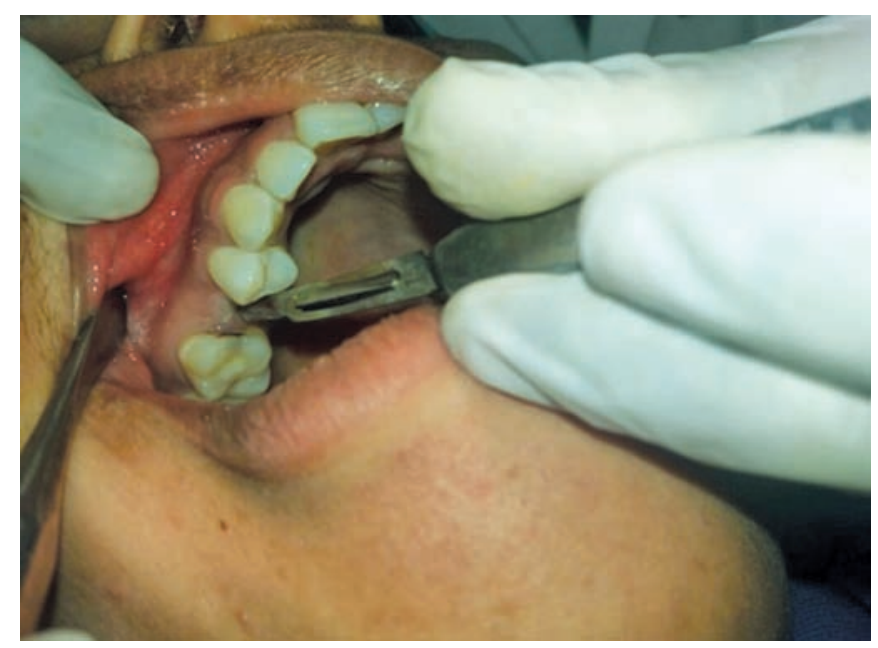

Fig. 2: Incision

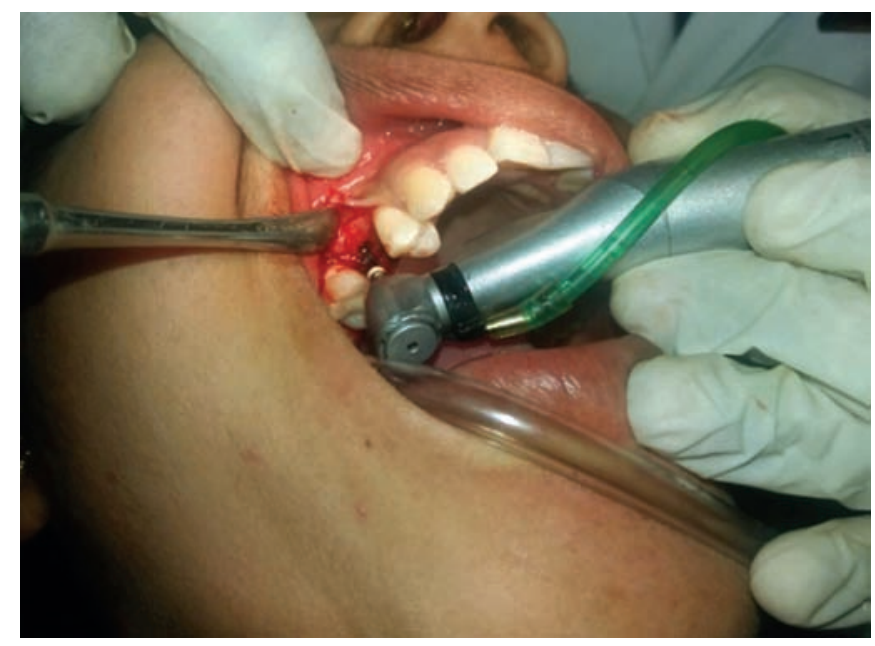

Fig. 4: Initial pilot drill

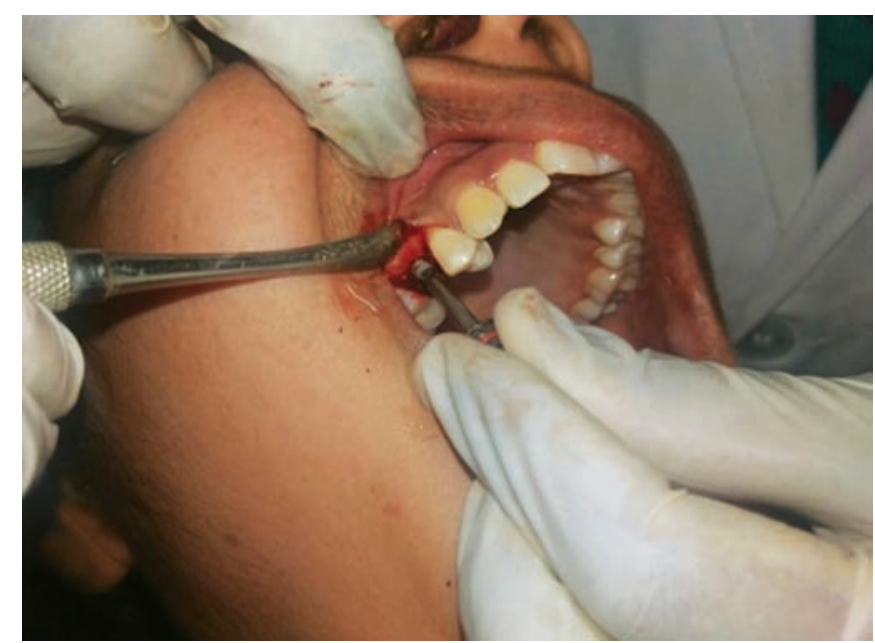

Fig. 6: Implant placement
- After final osteotomy, implant was driven at $20 \mathrm{rpm}$ and $50 \mathrm{Ncm}$ torque (Fig. 6).

- Cover screw was placed and suturing was done (Fig. 7).

- The sinus lift procedure was evaluated using an OPG (Fig. 8).

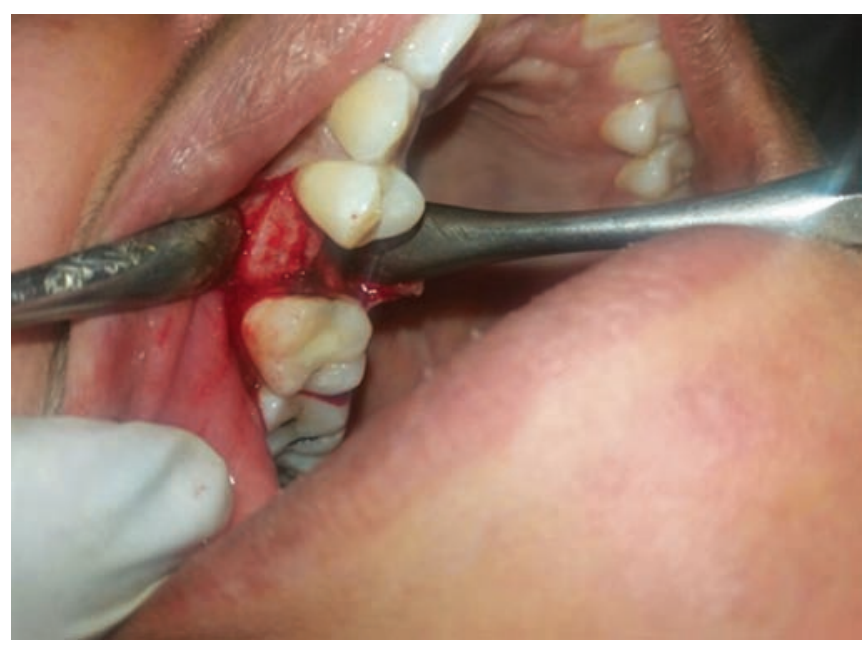

Fig. 3: Retraction

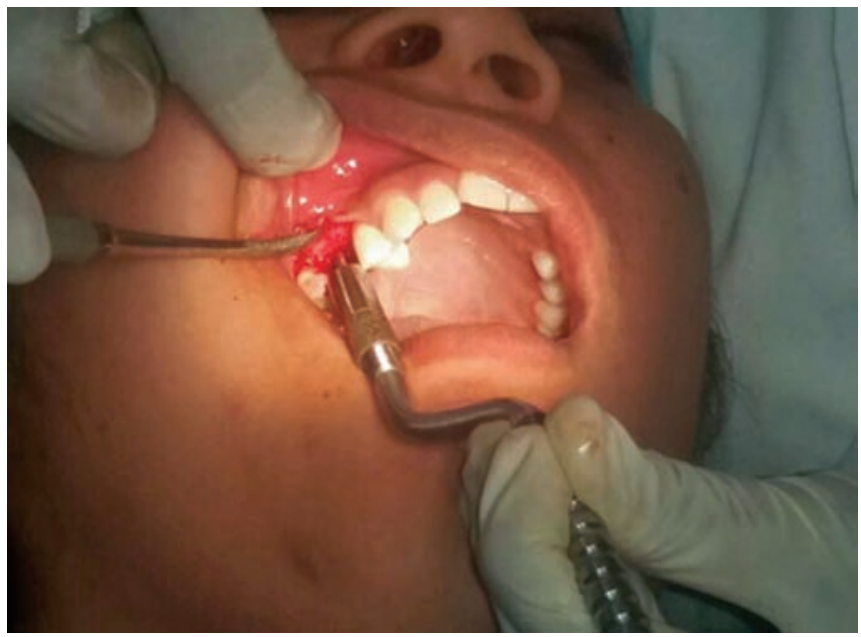

Fig. 5: Sinus lift instrumentation

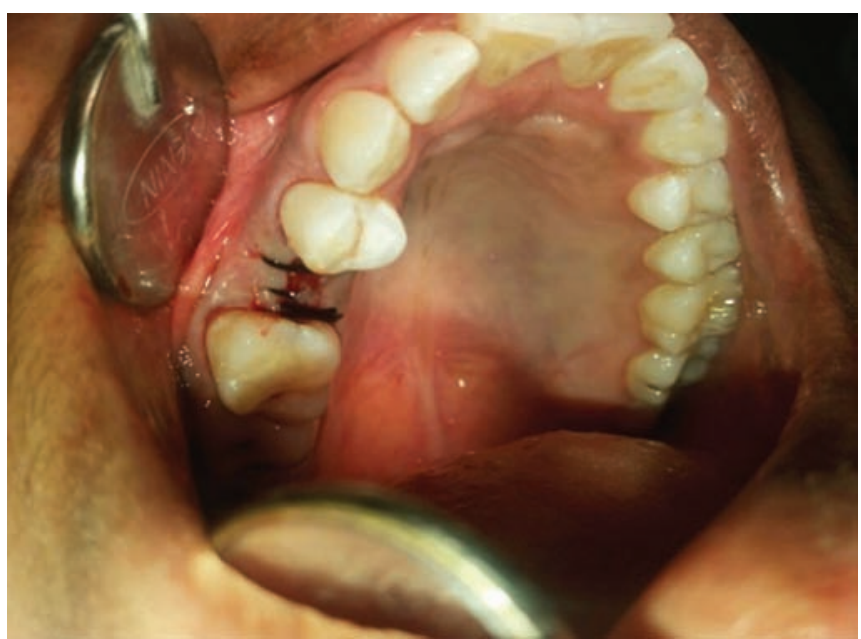

Fig. 7: Suturing 


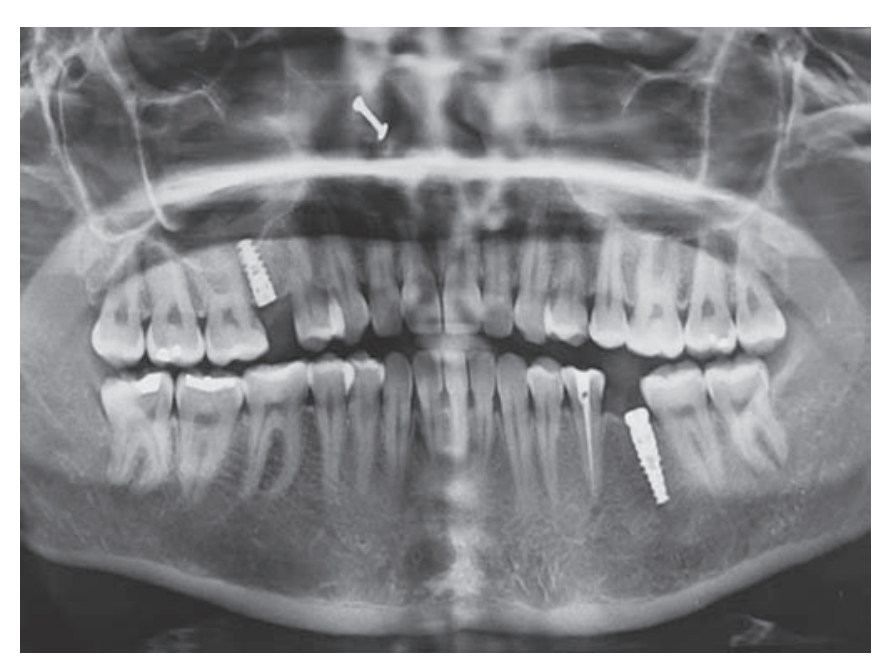

Fig. 8: Orthopantamograph post implant placement

\section{DISCUSSION}

With the advancement in dentistry, implant placement has become the most preferred means of replacement of missing teeth. In the posterior maxillary sextants, insertion of implants of desired length and diameter is often limited by the dimensional alterations of the residual ridge as well as the pneumatization of the maxillary sinus occurring after tooth loss. The maxilla is made up of spongy bone and has one of the least dense bones in oral cavity. To adapt, circumvent, and treat this local physiological as well as anatomical limitation; maxillary sinus floor elevation has become an important preplacement procedure in dental implant treatment planning. Various methodologies have evolved to increase the thickness of maxillary sinus floor. The treatment goal of all such procedures is to increase residual bone height. Few of the techniques involve simple, minimal elevation of maxillary sinus membrane, Schneiderian membrane, while others include placement of various types of grafts including allografts, autografts, bone morphogenetic proteins, and hydroxyapatite crystals. The most commonly used techniques are the direct sinus lift and indirect sinus lift. In the direct technique, the sinus is approached through the lateral window most of the time, whereas indirect technique follows a crestal approach. ${ }^{2}$ In this article, the indirect sinus lift approach using sinus elevation instruments has been followed for increasing the bone height and the bone width was increased using ridge splitting. ${ }^{3}$ The present case showed a bone height of $6.5 \mathrm{~mm}$ radiographically and the sinus floor was elevated up to $3.0 \mathrm{~mm}$ for the placement of implant of $9.5 \mathrm{~mm}$ length and $3.5 \mathrm{~mm}$ diameter. The study by showed a mean bone gain of $3 \mathrm{~mm}$ without any marginal bone loss after 3 to 12 months. ${ }^{4}$ The indirect sinus lift/ crestal approaches are minimally invasive but permit only a limited amount of augmentation. It is a highly predictable procedure, especially in the presence of minimal preoperative residual alveolar bone height. The crestal approach for sinus augmentation provides 97\% success rate, minimizing Schneiderian membrane perforation and the bone would form around the implant in 4 months and could be loaded. ${ }^{5}$ The disadvantage of this technique is that it is a blind procedure and only 2 to $3 \mathrm{~mm}$ of sinus elevation can be achieved.

\section{CONCLUSION}

The present case report illustrates a minimally invasive procedure aimed at sinus floor elevation for implant insertion. At times, placement of implants can be difficult due to reduced alveolar bone height and presence of anatomical defects, such as maxillary sinus. The indirect sinus lift procedure helps by keeping the technique simple and as predictable as possible to increase the success rate.

\section{REFERENCES}

1. Sani E, Veltri M, Cagidiaco MC, Balleri P, Ferrari M. Sinus membrane elevation in combination with placement of blasted implants: a 3-year case report of sinus augmentation without grafting material. Int J Oral Maxillofac Surg 2008 Oct;37(10):966-969.

2. Toffler M. Osteotome-mediated sinusfloor elevation: a clinical report. Int J Oral Maxillofac Implants 2004 Mar-Apr;19(2): 266-273.

3. Summers RB. A new concept in maxillary implant surgery: the osteotome technique. Compendium 1994 Feb;15(2):152-162.

4. Woo I, Le BT. Maxillary sinus floor elevation: review of anatomy and two techniques. Implant Dent 2004 Mar;13(1): 28-32.

5. Agnihotri A, Agnihotri D. Maxillary sinuses lift up: an indirect approach of implant placement in posterior maxilla. Int J Oral Implant Clin Res 2012 May-Aug;3(2):102-104. 